\title{
Improved LC-MS/MS method for the determination of 42 neurologically and metabolically important molecules in urine
}

\author{
Zsolt Galla *, Gábor Rácz, Nóra Grecsó, Ákos Baráth, Magdolna Kósa, Csaba Bereczki, \\ Péter Monostori \\ Metabolic and Newborn Screening Laboratory, Department of Paediatrics, University of Szeged
}

\section{A R T I C L E I N F O}

\section{Keywords:}

Kynurenine

Neurotransmitter

Pterin

LC-MS/MS

Urine

\begin{abstract}
A B S T R A C T
Simultaneous determination of kynurenines, neurotransmitters, pterins and steroids linked to various neurological and metabolic diseases have important diagnostic significance for related pathology and drug monitoring. An improved, sensitive and selective ultra-high performance liquid chromatography coupled to electrospray ionization triple quadrupole mass spectrometric (UHPLC-MS/MS) method, based on our earlier publication, has been proposed for the quantitative measurement of 42 metabolites in human urine. The assay covers a larger number of analytes, uses an advanced, Waters Atlantis T3 chromatographic column and similarly meets the guideline of European Medicines Agency (EMA) on bioanalytical method validation. Analytical performance met all the EMA requirements and the assay covered the relevant clinical concentrations. Linear correlation coefficients were all $>0.998$. Intra-day and inter-day accuracy and precision were $87-118 \%, 81-120 \%$ and $2-20 \%$, respectively including the lower limit of quantification (LLOQ). The assay is expected to facilitate the diagnosis and allows drug level monitoring from urine.
\end{abstract}

\section{Introduction}

Neurotransmitters, kynurenines and pterins play important role for various neurologic, metabolic and psychiatric disorders [1-3]. Concurrent measurement of these metabolites in human serum, cerebrospinal fluid (CSF) and urine helps to diagnose diverse diseases and gives opportunity to better understand differences in metabolic pathways. In addition, one can monitor complementary drug therapies on a broader spectrum. Not only the complementary drug levels can be measured, but the whole metabolic pathway, thus, the activity of metabolizing enzymes can also be examined (for example the determination of kynurenine (KYN)/triptophan (TRP) ratio suggest the indoleamine-2,3dioxigenase (IDO) and triptophan-2,3-deoxigenase (TDO) activity. Most laboratories use disease-specific methods which contain a few (3 1 0) metabolites [4-8] which make method development easier but limit the usability of the assay.

$95 \%$ of the tryptophan metabolism results in the formation of kynurenines. Differences in this metabolic pathway may play an important role in the course of diverse neurological diseases [9]. The remaining $5 \%$ of tryptophan is metabolized to serotonin and melatonin, which are essential for normal brain function [10]. The metabolic pathway starting from the amino acids phenylalanine and tyrosine (TYR) results in synthesis of several neurotransmitters that play a fundamental role in maintaining brain physiological function.

A quantitative determination of some diagnostically important molecules in human serum and CSF has already been presented in our previous study [11] based on the guideline of EMA on bioanalytical method validation [12]. Using this methodology we now aimed to develop an extended method for measuring urine samples using of an improved chromatographic separation and wider range of analytes. These include metanephrine (METANEP), normetanephrine (NORMETANEP), tyramine (TYRA), octopamine (OCT), phenetylamine (PEA), $N$ methylphenethylamine (NMPEA), quinaldic acid (QAA), picolinic acid (PA), methylserotonin (Me-5HT), dihydroneopterin (NH2), tetrahydrobiopterin (BH4), testosterone (TESTO), creatinine (CRN) and adenosine 3,5-cyclic monophosphate (cAMP). The determination of METANEP and NORMETANEP in addition to homovanillic acid (HVA) and vanillylmandelic acid (VMA) is a key laboratory test for the diagnosis of pheochromocytoma. NORMETANEP level is significantly elevated in patients treated with serotonin-norepinephrine reuptake

\footnotetext{
* Corresponding author at: Metabolic and Newborn Screening Laboratory, Department of Paediatrics, University of Szeged, Korányi fasor 14-15, H-6720 Szeged, Hungary.

E-mail address: galla.zsolt@med.u-szeged.hu (Z. Galla).
} 

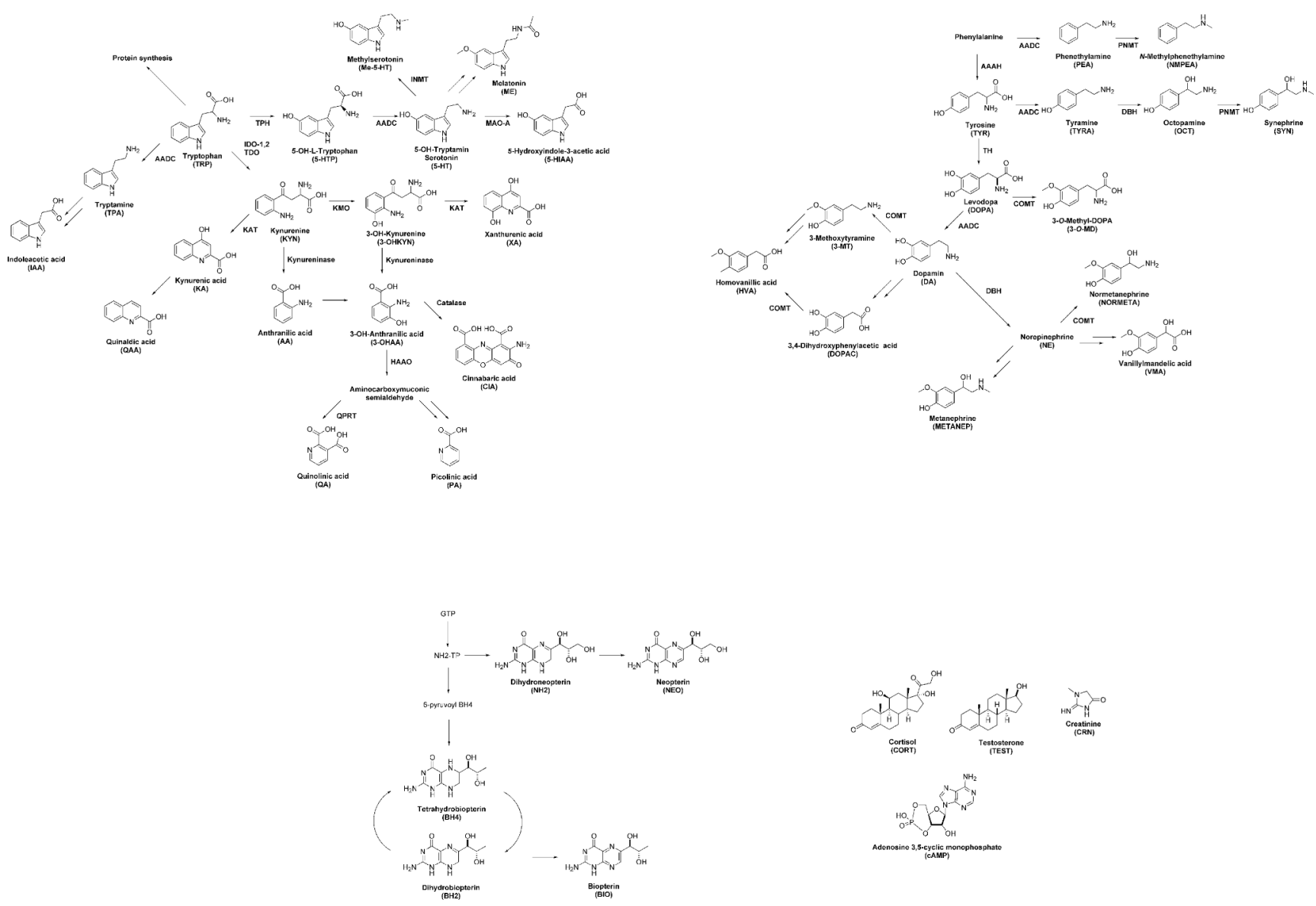

Fig. 1. Scheme of the 42 metabolites. TYR: tyrosine; DOPA: levodopa; 3-O-MD: 3-O-methyldopa; DA: dopamine; 3-MT: 3-methoxytyramine; DOPAC: 3,4-dihydroxyphenylacetic acid; HVA: homovanillic acid; VMA: vanillylmandelic acid; METANEP: metanephrine; NORMETANEP: normetanephrine; TYRA: tyramine; OCT: octopamine; SYN: synephrine; PEA: phenethylamine; NMPEA: N-methylphenethylamine; TRP: tryptophan; KYN: kynurenine; 3-OHK: 3-hydroxykynurenine; XA: xanthurenic acid; KA: kynurenic acid; QAA: quinaldic acid; AA: anthranilic acid; 3-OHAA: 3-hydroxyanthranilic acid; CIA: cinnabaric acid; QA: quinolinic acid; PA: picolinic acid; TPA: tryptamine; IAA: indole-3-acetic acid; 5-HTP: 5-hydroxy-tryptophan; 5-HT: serotonin; 5-HIAA: 5-hydroxyindolacetic acid; Me-5HT: methylserotonin; ME: melatonin; NH2: dihydroneopterin; NEO: neopterin; BH4: tetrahydrobiopterin; BH2: dihydrobiopterin; BIO: biopterin; CORT: cortisol; TESTO: testosterone; CRN: creatinine; cAMP: adenosine 3,5-cyclic monophosphate. TPH: tryptophan hydroxylase; AADC: aromatic amino acid decarboxylase; MAO-A: Monoamine oxidase-A; INMT: indolethylamine $N$-methyltransferase; IDO-1: indoleamine-2,3-dioxigenase-1; IDO-2: indoleamine-2,3-dioxigenase-2; TDO: triptophan-2,3-deoxigenase; KMO: kynurenine 3-monooxigenase; KAT: kynurenine aminotransferase; QPRT: quinolinate phosphoribosyl transferase; PNMT: phenylethanolamine $N$-methyltransferase; DBH: dopamine beta-hydroxylase; TH: tyrosine hydroxylase; COMT: catechol $O$-methyltransferase.

inhibitors (SNRI) or tricyclic antidepressants (TCAs) [13,14].

The separate measurement of the individual pterin species (biopterin (BIO), dihydrobiopterin (BH2), BH4, neopterin (NEO) and $\mathrm{NH} 2$ ) could help the differential diagnosis of tetrahydrobiopterin metabolism disorders (autosomal recessive guanosine triphosphate cyclohydrolase I (GTPCH) deficiency, 6-pyruvoyl tetrahydropterin synthase (PTPS) deficiency, dihydropteridine reductase (DHPR) deficiency, pterin-4$\alpha$-carbinolamine dehydratase (PCD) deficiency) (Fig. 1) [15,16].

Our aim was to develop a combined method to diagnose several diseases and monitor complementary drug therapies in a single run, such as DOPA (MADOPAR $®)$ or BH4 (KUVAN $®)$.

\section{Materials and methods}

\subsection{Chemicals and reagents}

Unlabelled standards as well as formic acid (FA), ascorbic acid, 1,4dithioerythritol (DTE), dimethyl sulfoxide (DMSO), Sigmatrix Urine Diluent and methanol $(\mathrm{MeOH})$ were purchased from Sigma (St. Louis, MO, USA) and acetonitrile (ACN) from Merck (Darmstadt, Germany). The solvents were LC-MS grade. The deuterated internal standard (IS) analogues DA-d4, TYR-d4 and DOPA-d3 were purchased from
Cambridge Isotope Laboratories (Andover, MA, USA), KA-d5, CRN-d3, CORT-d4, HVA-d5, NORMETANEP-d3 and METANEP-d3 from Sigma, QA-d3 from Buchem BV (Apeldoorn, Netherland) and 5-HT-d4 from Medical Isotopes Inc (Pelham, NH, USA). Steroids depleted serum was purchased from BBI Solutions (Crumlin, UK). Ultrapure water (18.2 M $\Omega$ $\times \mathrm{cm}$ ), filtered through a $0.22-\mu \mathrm{m}$ pore size membrane, was obtained from a Merck Millipore Direct-Q 3 UV system (Billerica, Mass., USA).

\subsection{Instrumentation}

The UHPLC-MS/MS system and MS parameters are the same as previously described [11], with the exception that a Waters Atlantis T3 $(2.1 \mathrm{~mm} \times 150 \mathrm{~mm}, 5 \mu \mathrm{m})$ column and an Atlantis T3 VanGuard Cartridge (Waters, Milford, MA, USA) were used for chromatographic analysis.

\subsection{UHPLC-MS/MS conditions}

For tuning, the analytes and IS in $0.2 \%$ FA were infused directly into the mass spectrometer and tuned for their molecular transitions in positive and/or negative ESI mode. Various percentages of acidified $(0.2 \% \mathrm{FA}) \mathrm{ACN} / \mathrm{H}_{2} \mathrm{O}$ and $\mathrm{MeOH} / \mathrm{H}_{2} \mathrm{O}$ have been tested in order to check 

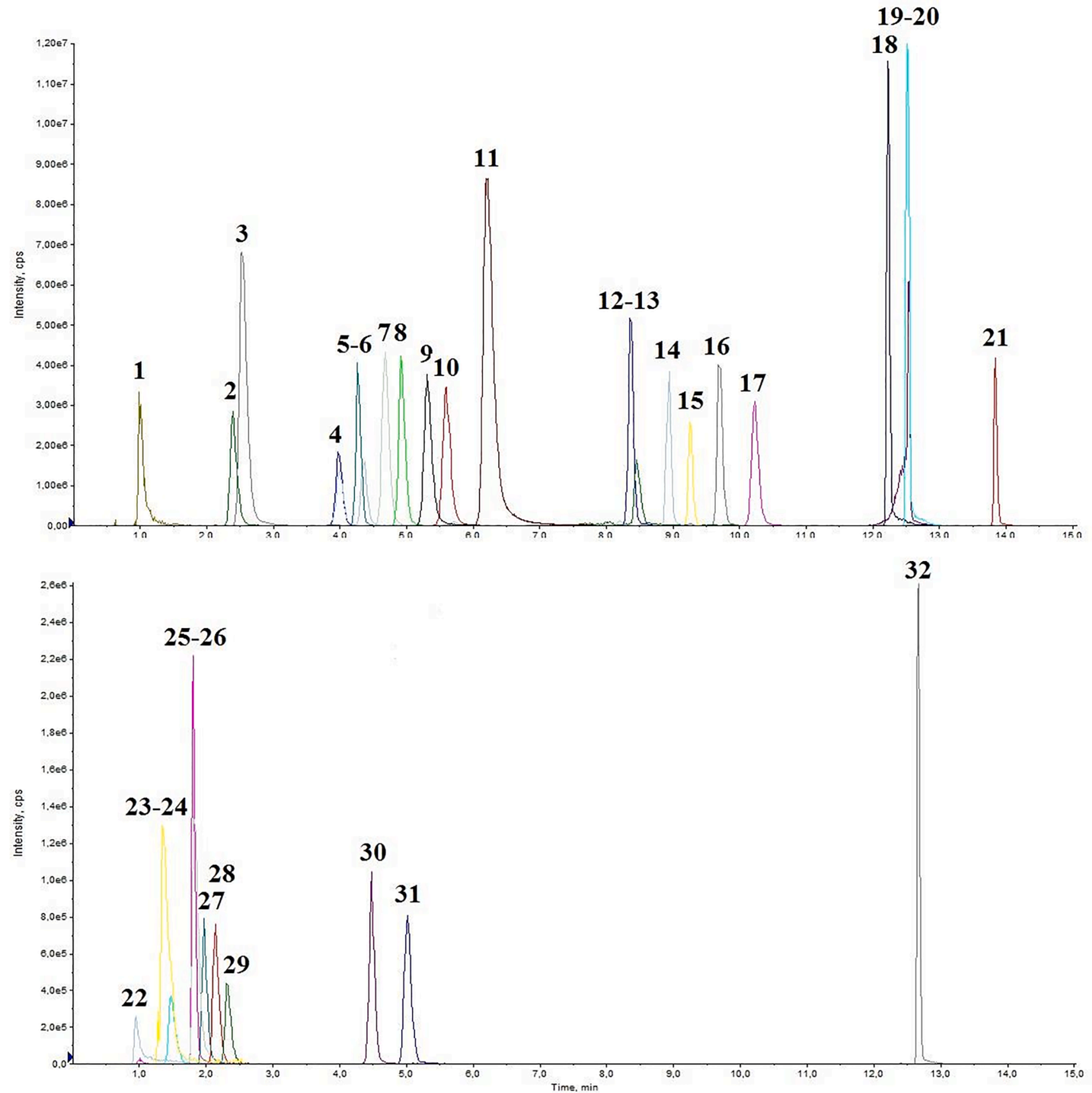

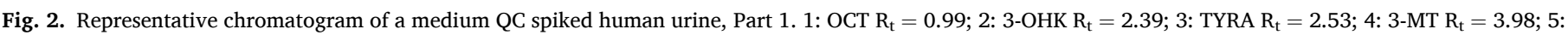

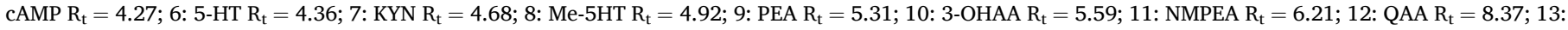

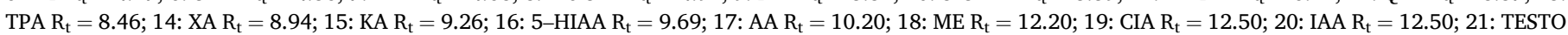

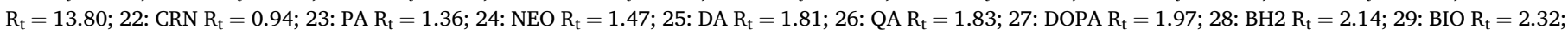
30: 3-O-MD $\mathrm{R}_{\mathrm{t}}=4.47 ; 31: 5$-HTP $\mathrm{R}_{\mathrm{t}}=5.01 ; 32$ : CORT $\mathrm{R}_{\mathrm{t}}=12.70$.

for molecule-specific ionization.

Mobile phase A consisted of ultrapure water plus 0.2\% FA (LC-MS grade). Mobile phase B consisted of ACN plus $0.2 \%$ FA (both LC-MS grade). Both eluents were prepared daily. The column temperature was $15{ }^{\circ} \mathrm{C}$, flow rate was kept at $0.6 \mathrm{~mL} / \mathrm{min}$ and the gradient profile was as follows: $0-1 \mathrm{~min}, 3 \% \mathrm{~B} ; 1-5.5 \mathrm{~min}, 3 \% \mathrm{~B}-7.5 \% \mathrm{~B}$; $5.5-8.3 \mathrm{~min}$, $7.5 \% \mathrm{~B}-16.5 \% \mathrm{~B} ; 8.3$ - $10 \mathrm{~min}, 16.5 \% \mathrm{~B}-23 \% \mathrm{~B} ; 10$ - $15 \mathrm{~min}, 23 \% \mathrm{~B}-$ $95 \%$ B; 15 - 16 min, 95\% B; 16 min - 17 min 95\% B - 3\% B; 17 min - 20 min $3 \%$ B. The slope of the gradient changes was linear. The injection volume was $15 \mu \mathrm{L}$ and the injection technique used was 'microliter pickup'. The sample compartment temperature was $10{ }^{\circ} \mathrm{C}$.

\subsection{Preparation of stock solutions}

Stock solutions and IS were prepared individually, according to their solubility and stability. Finally, the solutions were diluted with $\mathrm{H}_{2} \mathrm{O}$ / ACN/FA/ascorbic acid (96.9/3/0.2/0.02) (Diluting solution, DS) to two different mixtures (URINE MIX and IS MIX) according to their use (STable 1, STable 2). All standard stocks were stored at $-75^{\circ} \mathrm{C}$ in brown Eppendorf tubes in light protected containers. 

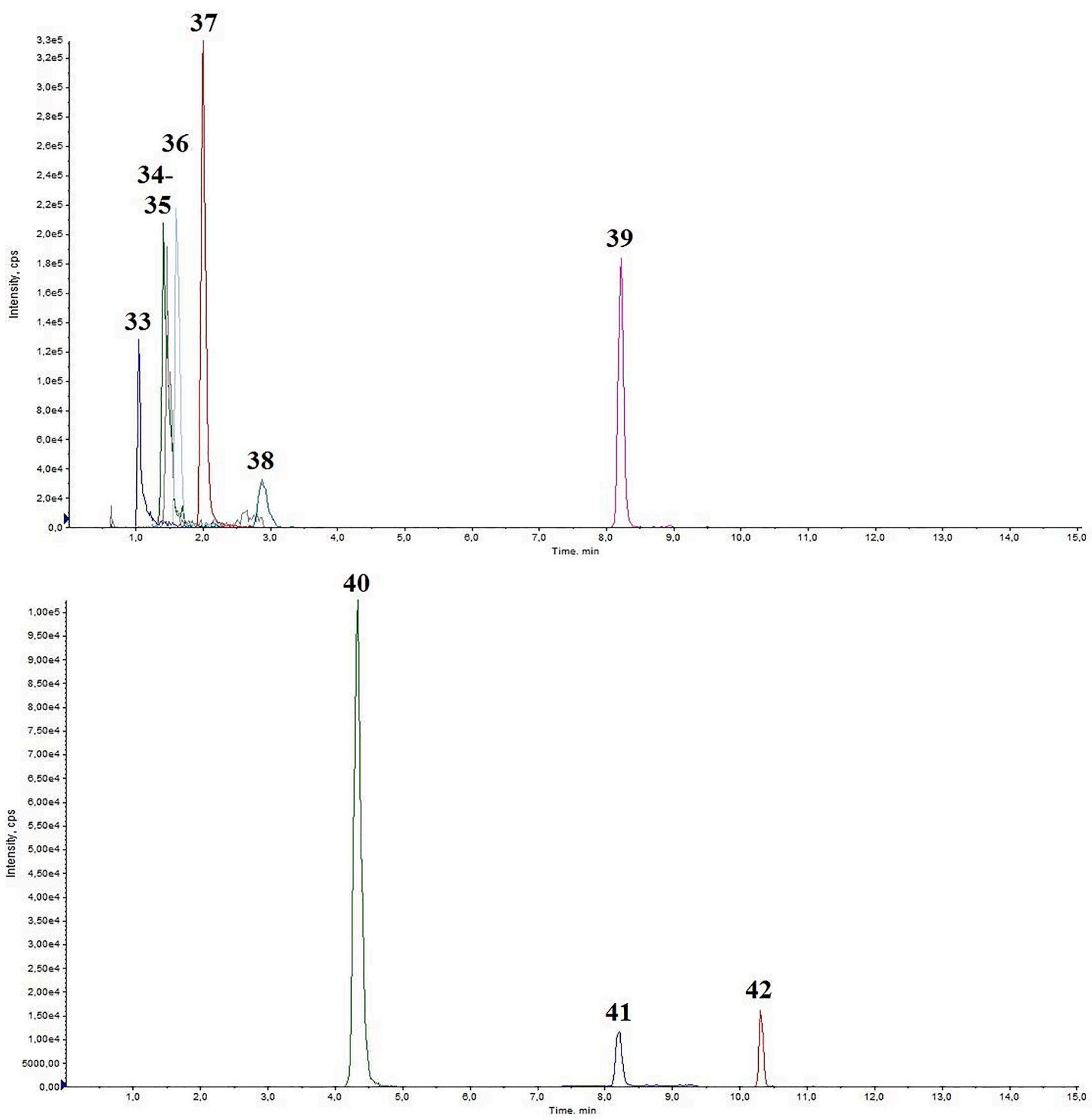

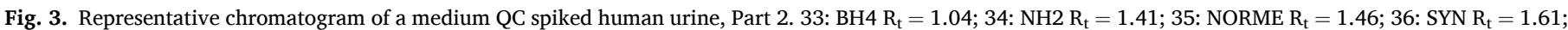

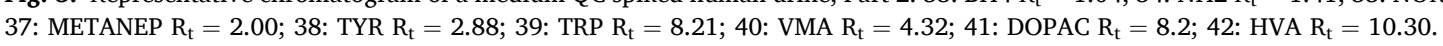

\subsection{Preparation of calibrators, quality controls (QC) and IS solutions}

The ranges of calibration standards were chosen to match the expected metabolite levels in human urine samples. The calibrators were prepared in a mixture of Sigmatrix Urine Diluent and $1 \mathrm{mg} / \mathrm{mL}$ DTE solution (1:3) and were spiked at nine concentration levels. The QCs were prepared as the calibrators but on different day and with different concentration levels as the calibrators and spiked at four levels of analytes (lower limit of quantitation (LLOQ), 3LLOQ, medium and high concentration).

A mixture of ISs was prepared by appropriate dilution of the stock solutions in DS (IS MIX). The calibrators, QCs and IS solutions were then aliquoted in brown Eppendorf tubes and stored at $-75^{\circ} \mathrm{C}$ in light protected containers until analysis.

\subsection{Collection and preparation of samples}

This study was approved by ethical committees (No. 139/2018-SZTE and IV/8004-1/2020/EKU). All patients participated voluntarily and gave their informed consent at the time of admission to the hospital for medical examinations, and for collecting urine samples.

Urine samples from 10 healthy volunteers (30-48 years old) were obtained for the validation of the method. The patient samples were used for diagnosis and medication monitoring in the Metabolic and Newborn Screening Laboratory in Szeged.

The urine samples were placed immediately on ice and centrifuged $\left(1000 \times \mathrm{g}\right.$ at $4{ }^{\circ} \mathrm{C}$ for $\left.10 \mathrm{~min}\right)$ within $60 \mathrm{~min} .90 \mu \mathrm{L} 1.0 \mathrm{mg} / \mathrm{mL}$ DTE solution was added to $30 \mu \mathrm{L}$ urine samples in a brown Eppendorf tube then stored at $-75{ }^{\circ} \mathrm{C}$ until analysis. 
Table 1

Creatinine normalized urine metabolite levels of 10 healthy volunteers and 5 treated PTPS patients.

\begin{tabular}{|c|c|c|}
\hline Analyte & $\begin{array}{l}\text { Control }(\mathrm{n}=10) \text { Mean } \pm \text { SD } \\
(\mathrm{nmol} / \mathrm{mmol} \mathrm{CRN})\end{array}$ & $\begin{array}{l}\text { PTPS patients }(\mathrm{n}=5) \text { Mean } \pm \\
\text { SD }(\mathrm{nmol} / \mathrm{mmol} \text { CRN) }\end{array}$ \\
\hline TYR & $15823 \pm 13904$ & $28655 \pm 15942$ \\
\hline DOPA & $26 \pm 23$ & $19892 \pm 24346$ \\
\hline 3-O-MD & $244 \pm 150$ & $63675 \pm 52304$ \\
\hline DA & $254 \pm 161$ & $24322 \pm 25277$ \\
\hline 3-MT & $89 \pm 71$ & $2588 \pm 1962$ \\
\hline DOPAC & $542 \pm 369$ & $31809 \pm 16209$ \\
\hline HVA & $4011 \pm 2048$ & $52914 \pm 18246$ \\
\hline VMA & $4006 \pm 2294$ & $1713 \pm 1212$ \\
\hline METANEP & $27 \pm 18$ & $16 \pm 9$ \\
\hline NORMETANEP & $26 \pm 21$ & $19 \pm 15$ \\
\hline TYRA & $331 \pm 192$ & $26 \pm 20$ \\
\hline OCT & $<$ LLOQ & $<$ LLOQ \\
\hline SYN & $4 \pm 3$ & $5 \pm 3$ \\
\hline PEA & $2 \pm 1$ & $1 \pm 0.5$ \\
\hline NMPEA & $<$ LLOQ & $<$ LLOQ \\
\hline TRP & $12783 \pm 7648$ & $14116 \pm 4659$ \\
\hline KYN & $747 \pm 409$ & $788 \pm 539$ \\
\hline 3-ОНК & $176 \pm 100$ & $256 \pm 133$ \\
\hline XA & $862 \pm 511$ & $320 \pm 92$ \\
\hline KA & $2022 \pm 1099$ & $624 \pm 195$ \\
\hline QAA & $16 \pm \overline{11}$ & $6 \pm 2$ \\
\hline AA & $13 \pm 11$ & $6 \pm 3$ \\
\hline 3-ОНАA & $727 \pm 434$ & $475 \pm 417$ \\
\hline CIA & $<$ LLOQ & $<$ LLOQ \\
\hline QA & $7258 \pm 5270$ & $5694 \pm 2014$ \\
\hline PA & $410 \pm 300$ & $249 \pm 117$ \\
\hline TPA & $117 \pm 84$ & $2 \pm 1$ \\
\hline IAA & $2889 \pm 2331$ & $3442 \pm 962$ \\
\hline 5-HTP & $20 \pm \overline{11}$ & $46 \pm \overline{31}$ \\
\hline 5-HT & $110 \pm 79$ & $50 \pm 12$ \\
\hline 5-HIAA & $1090 \pm 741$ & $245 \pm 95$ \\
\hline Me-5HT & $<$ LLOQ & $<$ LLOQ \\
\hline ME & $<$ LLOQ & $<$ LLOQ \\
\hline NH2 & $1055 \pm 708$ & $5201 \pm 3366$ \\
\hline NEO & $302 \pm 178$ & $928 \pm 212$ \\
\hline BH4 & $1061 \pm 762$ & $220 \pm 177$ \\
\hline BH2 & $525 \pm 185$ & $264 \pm 243$ \\
\hline BIO & $110 \pm 54$ & $107 \pm 71$ \\
\hline CORT & $13 \pm 4$ & $25 \pm 18$ \\
\hline TESTO & $0.8 \pm 0.6$ & $0.5 \pm 0.3$ \\
\hline сAMP & $673 \pm 352$ & $288 \pm 101$ \\
\hline
\end{tabular}

TYR: tyrosine; DOPA: levodopa; 3-O-MD: 3-O-methyldopa; DA: dopamine; 3MT: 3-methoxytyramine; DOPAC: 3,4-dihydroxyphenylacetic acid; HVA: homovanillic acid; VMA: vanillylmandelic acid; METANEP: metanephrine; NORMETANEP: normetanephrine; TYRA: tyramine; OCT: octopamine; SYN: synephrine; PEA: phenethylamine; NMPEA: N-methylphenethylamine; TRP: tryptophan; KYN: kynurenine; 3-OHK: 3-hydroxykynurenine; XA: xanthurenic acid; KA: kynurenic acid; QAA: quinaldic acid; AA: anthranilic acid; 3-OHAA: 3hydroxyanthranilic acid; CIA: cinnabaric acid; QA: quinolinic acid; PA: picolinic acid; TPA: tryptamine; IAA: indole-3-acetic acid; 5-HTP: 5-hydroxy-tryptophan; 5-HT: serotonin; 5-HIAA: 5-hydroxyindolacetic acid; Me-5HT: methylserotonin; ME: melatonin; NH2: dihydroneopterin; NEO: neopterin; BH4: tetrahydrobiopterin; BH2: dihydrobiopterin; BIO: biopterin; CORT: cortisol; TESTO: testosterone; CRN: creatinine; cAMP: adenosine 3,5-cyclic monophosphate, PTPS: 6-pyruvoyltetrahydropterin synthase.

To $120 \mu \mathrm{L}$ of DTE diluted urine $30 \mu \mathrm{L}$ of IS-MIX was added in a brown $1.5 \mathrm{~mL}$ Eppendorf tube. After vortexing for $4 \mathrm{sec}, 120 \mu \mathrm{L}$ was collected in a 96-well round-bottom microtiter plate (Corning 3365; Sigma (St. Louis, MO, USA)), sealed with aluminium foil and shaken for $30 \mathrm{~min}$. Fifteen $\mu \mathrm{L}$ was injected into the UHPLC-MS/MS system.

\subsection{Validation of the method}

For the validation of the urine analysis with UHPLC-MS/MS the following parameters were investigated: linearity, limit of detection (LOD), lower limit of quantitation (LLOQ), calibration curve, carry-over, intra and interday precision and accuracy, matrix effect, selectivity and stability. Recovery has not been calculated since the preparation of the samples contains only dilution steps. The validation process was evaluated according to the EMA guideline on bioanalytical methods [12] and as described in our previous study [11].

\section{Results \& discussion}

\subsection{Sample management}

Prolonged light, oxygen or temperature exposure is critical for the stability of the measured analytes $[17,18]$. For this reason, the urine samples were transported to the laboratory on ice in light protected container after collection. The samples were centrifuged (3000 RPM, $4{ }^{\circ} \mathrm{C}$ for $\left.10 \mathrm{~min}\right)$. The supernatants were diluted with $1 \mathrm{mg} / \mathrm{mL}$ DTE solution (1:3) then aliquoted and stored at $-75{ }^{\circ} \mathrm{C}$ until use.

\subsection{LC-MS/MS optimization}

The UHPLC-MS/MS parameters and the preliminary experiments of LC and MS were the same as described previously [11]. The Waters Atlantis dc18 column was also tested here, but the chromatographic challenges set by the addition of further biomarkers and the use of urine specimens could only be addressed by the T3 column. MS parameters, polarities, retention times, related ISs and quantifiers are shown in STable 3. The representative chromatograms of medium QC spiked human urine are shown in the Supplementary material (Figs. 2-3).

\subsection{Method validation}

\subsubsection{Linearity, $L O D, L L O Q$ and calibration curve.}

Linearity, LOD and LLOQ were determined in a mixture of $1 \mathrm{mg} / \mathrm{mL}$ DTE, Sigmatrix Urine Diluent and IS MIX (3:1:1) then $15 \mu \mathrm{L}$ was injected to the UHPLC-MS/MS system. LOD and LLOQ were calculated using the Analyst 1.6.2. software. The LLOQ represented the lowest concentration where the desired accuracy and precision were met. The determination coefficients were excellent $\left(R^{2}: 0.998-1.000\right)$. The calibration curves contained at least seven calibrator points for each analyte. The results are shown in STable 4.

\subsubsection{Carry-over}

Carry-over was investigated by injecting three different, high-level spiked human urine (prepared from $30 \mu \mathrm{L}$ urine $(0.96 \mathrm{mM}$ creatinine; $3.1 \mathrm{mM}$ creatinine and $5.0 \mathrm{mM}$ creatinine), $30 \mu \mathrm{L}$ URINE MIX and $90 \mu \mathrm{L}$ $1.0 \mathrm{mg} / \mathrm{mL}$ DTE solution) and analyte-free DS alternately. After each injection, 5 needle wash cycles with DS were used to eliminate carryover, which was defined as blank samples displaying peaks with a height $>1 \%$ of the peak of the corresponding peak. Carry-over was less than $0.5 \%$ for all analytes and did not exceed $15 \%$ of the value of the area of LLOQ.

\subsubsection{Precision and accuracy}

Intra-day accuracy and precision was determined by analysing 10 URINE QC samples at four concentration levels in a single run (LLOQ, 3LLOQ, medium and high spiked QCs) while interday accuracy and precision was determined in $5 \mathrm{QC}$ samples at four concentration levels in three different days (STable 5). According to EMA regulation [12], accuracies were acceptable when the mean concentrations were within $15 \%$ of the nominal values (except for the LLOQ spiked samples where $\leq 20 \%$ ) and precision was satisfactory when the RSD values $\leq 15 \%$ (LLOQ: $\leq 20 \%$ ). All analytes met the requirements.

\subsubsection{Matrix effect, recovery and selectivity.}

Matrix effects were investigated in two different human urine specimens (QCM_1: CRN 0.96 mM; QCM_2: CRN $3.29 \mathrm{mM}$ ), spiked at two levels (medium and high QC) and measured five times in a row. Matrix factor (MF) was calculated for each analyte as the ratio of the analyte/IS 

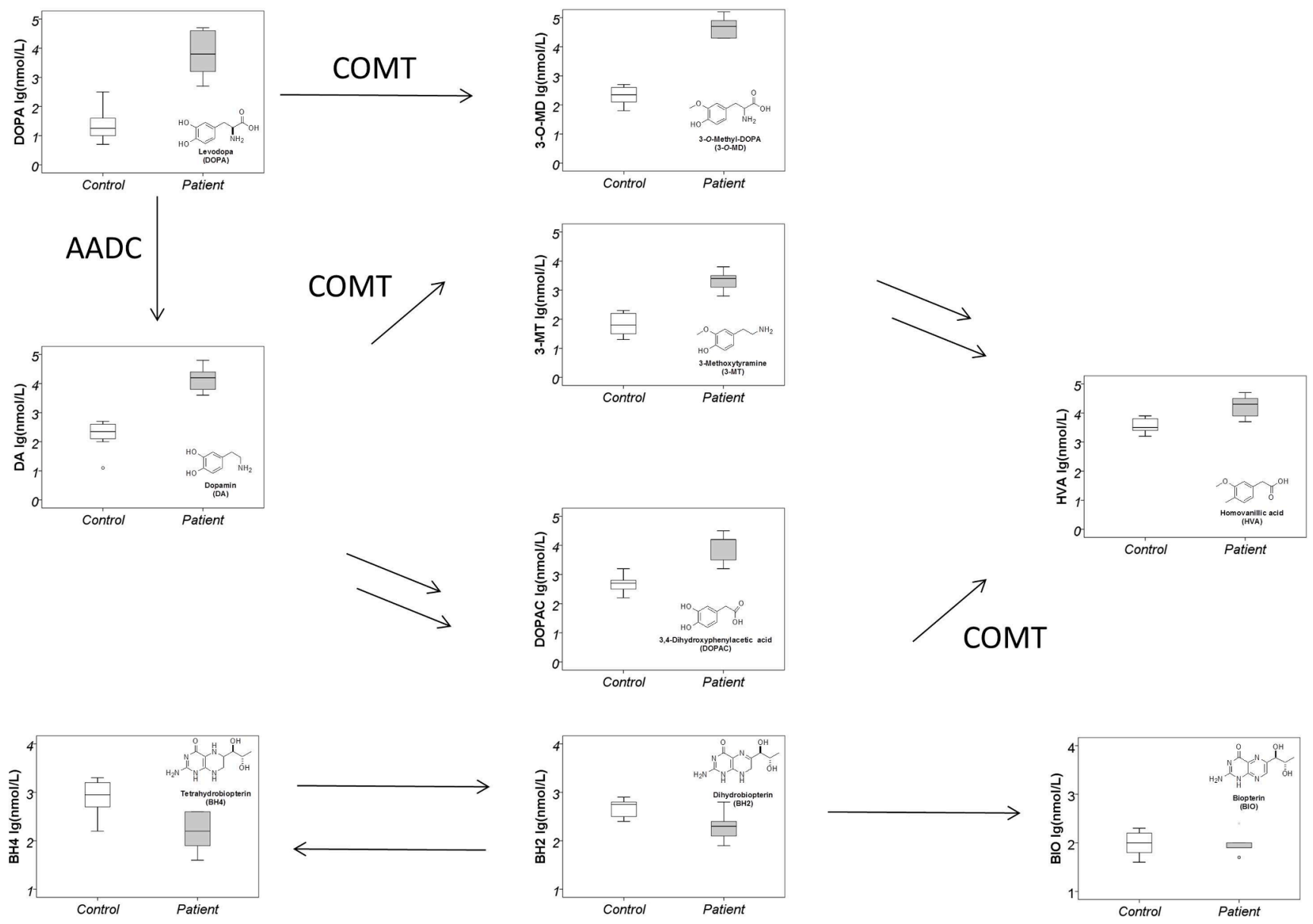

Fig. 4. Creatinine normalized lg values of DOPA, 3-O-MD, DA, DOPAC, 3-MT, HVA, BH4, BH2 and BIO of 5 DOPA and BH4 treated PTPS patients and 10 non-treated healthy patients. Results are presented in an arrangement that corresponds to the biochemical routes. DOPA: levodopa; 3-O-MD: 3-O-methyldopa; DA: dopamine; 3-MT: 3-methoxytyramine; DOPAC: 3,4-dihydroxyphenylacetic acid; HVA: homovanillic acid; NH2: dihydroneopterin; NEO: neopterin; BH4: tetrahydrobiopterin; BH2: dihydrobiopterin; BIO: biopterin; AADC: aromatic amino acid decarboxylase; COMT: catechol $O$-methyltransferase.

peak area in the presence of matrix divided by the ratio analyte/IS peak area in post-extraction samples without matrix, multiplied by $100 \%$. MF was regarded as acceptable when the $\mathrm{CV}$ of $\mathrm{MF} \leq 15 \%$. (STable6).

No significant endogenous matrix interferences were observed and there were no noticeable co-eluting compounds in the human urine samples.

\subsubsection{Stability}

The stability of the compounds was investigated at 3LLOQ and in the high QC at different storage conditions in different matrices. The compounds were regarded stable when recovery and accuracy was $100 \pm$ $20 \%$.

Stock solutions, urine samples were stable at least $24 \mathrm{~h}$ at $4{ }^{\circ} \mathrm{C}$ and 3 months at $-80^{\circ} \mathrm{C}$. Prepared urine samples were stable in the autosampler $\left(10^{\circ} \mathrm{C}\right)$ at least for $24 \mathrm{~h}$. Stability tests after 3 freeze-thaw cycles showed no decomposition.

\subsection{Evaluation of blank matrix}

The blank matrix (Sigmatrix Urine Diluent) was injected ten times in the UHPLC-MS/MS system. There were no interferences with the measured analytes and the blank matrix except for CRN which is in the Sigmatrix Urine Diluent. Besides that before every measurement 1 blank matrix was injected into the UHPLC-MS/MS system.

\subsection{CRN measurement and CRN normalized metabolite levels}

In the routine diagnostics, urinary metabolite levels normalized to $\mathrm{CRN}$ are more appropriate than quantifying analytes in absolute units (e. g. $\mathrm{ng} / \mathrm{mL}$ or $\mathrm{nmol} / \mathrm{L}$ ) in compensating differential urinary concentrations [19]. The classical method for the determination of urinary creatinine is the Jaffe's reaction, where creatinine produces quantitatively an orange colour with picric acid in alkaline medium [20]. CRN determined with the same UHPLC-MS/MS method as the measured analytes can shorten sample preparation time.

\subsection{Application of the method: Healthy human samples and substitution drug therapy monitoring measurements}

Of potential options to prove the clinical effectiveness of the method (e.g. diagnosis of BH4 deficiency [21], aromatic L-amino acid decarboxylase deficiency [22], neuroblastoma [23] etc.), therapy monitoring of 6-pyruvoyltetrahydropterin synthase deficiency (PTPS) patients was chosen. Substitution treatment with neurotransmitter precursors such as DOPA and BH4 result in marked elevation of certain analytes (3-O-MD, DA, DOPAC, 3-MT, HVA, BH2 and BIO) in the metabolic pathway which makes their analytical determination challenging. Our calibration ranges cover metabolite levels in health and disease for a reliable clinical diagnosis but analyte levels during substitution therapy can be outside of these ranges. Thus, urine samples of patients receiving adjunctive therapy was diluted 10 - and 30 -fold before the sample preparation. In PTPS due to the malfunction of the 6-pyruvoyltetrahydropterin synthase 
enzyme the production of $\mathrm{BH} 4, \mathrm{BH} 2$ and $\mathrm{BIO}$ is decreased while NEO and $\mathrm{NH} 2$ increased. $\mathrm{BH} 4$ is an essential cofactor of vital enzymes (phenylalanine hydroxylase, tyrosine hydroxylase, tryptophan hydroxylase, alkylglycerol monooxygenase and nitric oxide synthase) which makes DOPA treatment also necessary. Table 1 contains the CRN normalized urinary levels in ten healthy controls and five DOPA and BH4 threated PTPS patients. Despite the treatment, it was shown that the NEO and NH2 urinary levels in the PTPS patients were still elevated and $\mathrm{BH} 4, \mathrm{BH} 2$ and BIO levels did not reach the values of the control group. Examination of the treatment is shown in Fig. 4, where the logarithmical values of metabolite concentrations are plotted for better comparability.

\section{Conclusion}

An improved, sensitive and selective UHPLC-MS/MS method, based on our earlier publication has been developed and validated for the quantitative measurement of TYR and TRP and their metabolites, pterins and steroids in human urine. The adequacy of the method has been proved through the guideline of EMA on bioanalytical method validation. The new method can be used to extend the quantitative study of metabolic pathways. Using new column and modified gradient allows to measure 42 analytes instead of 30 . From a clinical point of view, the method is suitable for making a diagnosis for some metabolic and neurological several diseases and allows drug level monitoring from urine. The suitability of the method for measuring serum and CSF samples should be investigated in the future.

\section{CRediT authorship contribution statement}

Zsolt Galla: Conceptualization, Investigation, Methodology, Validation, Formal analysis, Writing - original draft. Gábor Rácz: Conceptualization, Resources, Writing - review \& editing. Nóra Grecsó: Conceptualization, Resources, Writing - review \& editing. Ákos Baráth: Conceptualization, Resources, Writing - review \& editing. Magdolna Kósa: Conceptualization, Resources, Writing - review \& editing. Csaba Bereczki: Resources, Writing - review \& editing, Supervision. Péter Monostori: Conceptualization, Investigation, Resources, Writing - review \& editing, Project administration.

\section{Declaration of Competing Interest}

The authors declare that they have no known competing financial interests or personal relationships that could have appeared to influence the work reported in this paper.

\section{Acknowledgement}

Anita Zádori for the technical assistance in sample preparations. Article Processing Charges were covered by the Institutional Account Program of the University of Szeged, Szeged, Hungary (Open Access Fund, Grant number: 5196). The funder had no role in study design, data collection and analysis, decision to publish, or preparation of the manuscript.

\section{Appendix A. Supplementary material}

Supplementary data to this article can be found online at https://doi. org/10.1016/j.jchromb.2021.122846.

\section{References}

[1] D.C. Maddison, F. Giorgini, The kynurenine pathway and neurodegenerative disease, Seminars in cell \& developmental biology 40 (2015) 134-141, https://doi. org/10.1016/j.semcdb.2015.03.002.

[2] J.-M.S.G.v.d.B.J.H. Walter, Inborn Metabolic Diseases, fourth ed., Springer, 2012.
[3] S. Jung-Klawitter, O. Kuseyri Hübschmann, Analysis of Catecholamines and Pterins in Inborn Errors of Monoamine Neurotransmitter Metabolism-From Past to Future, Cells 8 (2019) 867.

[4] H. Tomšíková, P. Solich, L. Nováková, Sample preparation and UHPLC-FD analysis of pteridines in human urine, Journal of Pharmaceutical and Biomedical Analysis 95 (2014) 265-272, https://doi.org/10.1016/j.jpba.2014.03.012.

[5] Z.D. Clark, E.L. Frank, Urinary metanephrines by liquid chromatography tandem mass spectrometry: Using multiple quantification methods to minimize interferences in a high throughput method, Journal of Chromatography B 879 (2011) 3673-3680, https://doi.org/10.1016/j.jchromb.2011.10.005.

[6] Z. Xie, P. Lorkiewicz, D.W. Riggs, A. Bhatnagar, S. Srivastava, Comprehensive, robust, and sensitive UPLC-MS/MS analysis of free biogenic monoamines and their metabolites in urine, Journal of chromatography, B, Analytical technologies in the biomedical and life sciences 1099 (2018) 83-91, https://doi.org/10.1016/j. jchromb.2018.09.012.

[7] K. Syslová, L. Rambousek, M. Kuzma, V. Najmanová, V. Bubeníková-Valešová, R. Šlamberová, P. Kačer, Monitoring of dopamine and its metabolites in brain microdialysates: Method combining freeze-drying with liquid chromatography-tandem mass spectrometry, Journal of Chromatography A 1218 (2011) 3382-3391, https://doi.org/10.1016/j.chroma.2011.02.006.

[8] P. Guibal, N. Lévêque, D. Doummar, N. Giraud, E. Roze, D. Rodriguez, R. Couderc, T. Billette De Villemeur, F. Moussa, Simultaneous determination of all forms of biopterin and neopterin in cerebrospinal fluid, ACS Chem Neurosci 5 (2014) 533-541, https://doi.org/10.1021/cn4001928.

[9] R. Schwarcz, J.P. Bruno, P.J. Muchowski, H.-Q. Wu, Kynurenines in the mammalian brain: when physiology meets pathology, Nat Rev Neurosci 13 (2012) 465-477, https://doi.org/10.1038/nrn3257.

[10] R.L. Carhart-Harris, D.J. Nutt, Serotonin and brain function: a tale of two receptors, J Psychopharmacol 31 (2017) 1091-1120, https://doi.org/10.1177/ 0269881117725915.

[11] Z. Galla, C. Rajda, G. Rácz, N. Grecsó, Á. Baráth, L. Vécsei, C. Bereczki, P. Monostori, Simultaneous determination of 30 neurologically and metabolically important molecules: A sensitive and selective way to measure tyrosine and tryptophan pathway metabolites and other biomarkers in human serum and cerebrospinal fluid, Journal of chromatography. A 1635 (2021), 461775, https:// doi.org/10.1016/j.chroma.2020.461775.

[12] Guideline on bioanalytical method validation. European Medicines Agency Reference number: EMEA/CHMP/EWP/192217/2009 Rev. 1 Corr. 2. Effective from: February 1st 2012. Available from: https://www.ema.europa.eu/en/ bioanalytical-method-validation in, 2020.

[13] J.W.M. Lenders, G. Eisenhofer, Normetanephrine and Metanephrine, in: I. Huhtaniemi, L. Martini (Eds.) Encyclopedia of Endocrine Diseases (Second Edition), Academic Press, Oxford, 2017, pp. 420-424, https://doi.org/10.1016/ B978-0-12-801238-3.03975-1.

[14] G. Kline, A.C. Chin, Chapter 5 - Adrenal disorders, in: H. Sadrzadeh, G. Kline (Eds.), Endocrine Biomarkers, Elsevier (2017) 181-249, https://doi.org/10.1016/B978-012-803412-5.00005-7.

[15] N. Blau, L. Bonafé, B. Thöny, Tetrahydrobiopterin Deficiencies without Hyperphenylalaninemia: Diagnosis and Genetics of DOPA-Responsive Dystonia and Sepiapterin Reductase Deficiency, Molecular Genetics and Metabolism 74 (2001) 172-185, https://doi.org/10.1006/mgme.2001.3213.

[16] N. Blau, J.B. Hennermann, U. Langenbeck, U. Lichter-Konecki, Diagnosis, classification, and genetics of phenylketonuria and tetrahydrobiopterin (BH4) deficiencies, Molecular Genetics and Metabolism 104 (2011) S2-S9, https://doi. org/10.1016/j.ymgme.2011.08.017.

[17] X. Yang, Y. Hu, G. Li, Online micro-solid-phase extraction based on boronate affinity monolithic column coupled with high-performance liquid chromatography for the determination of monoamine neurotransmitters in human urine, Journal of Chromatography A 1342 (2014) 37-43, https://doi.org/10.1016/j. chroma.2014.03.041.

[18] B. stea, R.M. Halpern, B.C. Halpern, R.A. Smith, Quantitative determination of pterins in biological fluids by high-performance liquid chromatography, Journal of Chromatography A 188 (1980) 363-375, https://doi.org/10.1016/S0021-9673 (00)81259-5.

[19] K.W.A. Tang, Q.C. Toh, B.W. Teo, Normalisation of urinary biomarkers to creatinine for clinical practice and research-when and why, Singapore Med J 56 (2015) 7-10, https://doi.org/10.11622/smedj.2015003.

[20] B.D. Toora, G. Rajagopal, Measurement of creatinine by Jaffe's reaction-determination of concentration of sodium hydroxide required for maximum color development in standard, urine and protein free filtrate of serum, Indian journal of experimental biology 40 (2002) 352-354.

[21] T. Opladen, E. López-Laso, E. Cortès-Saladelafont, T.S. Pearson, H.S. Sivri, Y. Yildiz, B. Assmann, M.A. Kurian, V. Leuzzi, S. Heales, S. Pope, F. Porta, A. GarcíaCazorla, T. Honzík, R. Pons, L. Regal, H. Goez, R. Artuch, G.F. Hoffmann, G. Horvath, B. Thöny, S. Scholl-Bürgi, A. Burlina, M.M. Verbeek, M. Mastrangelo, J. Friedman, T. Wassenberg, K. Jeltsch, J. Kulhánek, O. Kuseyri Hübschmann, D. on behalf of the International Working Group on Neurotransmitter related, Consensus 
guideline for the diagnosis and treatment of tetrahydrobiopterin $(\mathrm{BH} 4)$ deficiencies, Orphanet J Rare Dis, 15 (2020) 126, 10.1186/s13023-020-01379-8.

[22] T. Wassenberg, M. Molero-Luis, K. Jeltsch, G.F. Hoffmann, B. Assmann, N. Blau, A. Garcia-Cazorla, R. Artuch, R. Pons, T.S. Pearson, V. Leuzzi, M. Mastrangelo, P. L. Pearl, W.T. Lee, M.A. Kurian, S. Heales, L. Flint, M. Verbeek, M. Willemsen,

T. Opladen, Consensus guideline for the diagnosis and treatment of aromatic 1 - amino acid decarboxylase (AADC) deficiency, Orphanet J Rare Dis 12 (2017) 12, https://doi.org/10.1186/s13023-016-0522-z.

[23] C.C. Swift, M.J. Eklund, J.M. Kraveka, A.L. Alazraki, Updates in Diagnosis, Management, and Treatment of Neuroblastoma, RadioGraphics 38 (2018) 566-580, https://doi.org/10.1148/rg.2018170132. 